\title{
Effect of the Morphology of Leather Surface on the Hydrophobic-Hydrophilic Properties
}

\author{
Olga Serenko ${ }^{1}$, Zarina Nizamova², Mikhail Kalinin², Yuri Ostrovsky², \\ Ludmila Polukhina ${ }^{2}$, Aziz Muzafarov ${ }^{1}$ \\ ${ }^{1}$ Enikolopov Institute of Synthetic Polymeric Materials, \\ Foundation of the Russian Academy of Sciences, Moscow, Russia \\ ${ }^{2}$ Moscow State University of Design and Technology, Moscow, Russia \\ Email: oserenko@yandex.ru, znizamova@mail.ru, mgudt.mal@mail.ru, ostrovsky_y@bk.ru, artek39@mail.ru, aziz@ispm.ru
}

Received December 25, 2013; revised January 24, 2014; accepted February 6, 2014

Copyright (C) 2014 Olga Serenko et al. This is an open access article distributed under the Creative Commons Attribution License, which permits unrestricted use, distribution, and reproduction in any medium, provided the original work is properly cited. In accordance of the Creative Commons Attribution License all Copyrights (C) 2014 are reserved for SCIRP and the owner of the intellectual property Olga Serenko et al. All Copyright (C) 2014 are guarded by law and by SCIRP as a guardian.

\begin{abstract}
Natural leathers (split, velour split, nubuck) ware investigated. Solution of fluorine silane in ethanol was applied as the hydrophobic agent. It is shown that the microstructure of the leather surface, provided its structural elements possess hydrophilic properties, determines the wetting regime and duration of the induction period of changeover from heterogeneous to homogeneous wetting. The heterogeneous regime of wetting is distinguished by low rates of spreading and absorbing. The homogeneous wetting is characterized by high rates of spreading and absorbing of a water drop. A necessary condition of the stable heterogeneous wetting regime and achieving hydrophobicity of leather with structurally inhomogeneous face layer is to preserve the microstructure features of their surface at hydrophobic treatment. The morphological peculiarities of split and ground leather ensure stable hydrophobic properties after application of efficient hydrophobizators.
\end{abstract}

\section{KEYWORDS}

Leather; Surface Modification; Water Contact Angle; Wetting; Hydrophobic Effect

\section{Introduction}

The production of comfortable waterproof shoes for autumn, spring and winter involves the use of watertight leather, which satisfies the exploitational, hygienic and esthetic requirements put to the materials of which the shoe uppers are made. To obtain leather with water-proof properties, it is supposed to apply thin polymeric layer to its surface [1-5] or the use of a polymer composition containing nanoscale particles [6-9] at the finishing step of processing. Materials produced in this manner can be classified as leather with a smooth upper surface [1].

In contrast, the surface of polished leather or split leather characterized by a certain roughness is extremely heterogeneous. These circumstances, coupled with capillary-porous morphology of the surface layer of these skins, should be considered when creating hydrophobic materials based on them.

Wetting structurally inhomogeneous surface of leather, the mechanisms of spreading and absorption of water droplets will largely depend on the hydrophilicity of the individual elements of its structure (capillaries, pores, collagen, etc.). Additionally, surface irregularities and, as a result, air contained in the surface layer, are factors, which contribute to the heterogeneous regime wetting (the wetting model of Cassie-Baxter). The latter refers to one of the necessary conditions for achieving the hydrophobicity of the surface [10-12]. Investigation of leather morphology with a rough, heterogeneous facial layer and its influence on the hydrophobic-hydrophilic properties will set the peculiarities of the formation of water-repellent characteristics and conditions of hydrophobic treatment.

Present study deals with the kinetics of wetting, spreading and absorption of water droplets to determine the impact of the surface morphology of initial and hydrophobized leather on its hydrophilic-hydrophobic properties. 


\section{Materials and Methods}

The materials in this work were several kinds of finished natural shoe upper leathers: split, velour split and nubuck (Russia). 5\% solution of 2,2,3,3,4,4,5,5,6,6,7,7,8,8,9,9,9heptadecafluor-N-[3-triethyoxysilyl)propyl]-nonanamide (fluorine silane) in ethanol was used as a hydrophobic agent. The structural formula of this modifier is as follows, $\mathrm{CF}_{3}-\left(\mathrm{CF}_{2}\right)_{7}-\mathrm{C}(\mathrm{O})-\mathrm{NH}-\left(\mathrm{CH}_{2}\right)_{3}-\mathrm{Si}\left(\mathrm{OC}_{2} \mathrm{H}_{5}\right)_{3}$ High efficiency of this compound for hydrophobization of textil, leather and fur was demonstrated in paper [13, 14].

The surface treatment of leather was conducted by spraying the solution perpendicular to the material surface from $20 \mathrm{~cm}$ above it with follow-on exposure of the specimens at temperature $20^{\circ} \mathrm{C}-25^{\circ} \mathrm{C}$ for $20 \mathrm{~min}$ and then for $10 \mathrm{~min}$ at $90^{\circ} \mathrm{C}$. The need in additional thermal treatment was substantiated earlier in [13]. The average consumption of the $5 \%$-solution of hydrophobizator was $0.4 \mathrm{ml} / \mathrm{dm}^{2}$.

The water contact angle $\Theta$ and changes in the drop contour were determined by analysis of video images of drop spreading and absorption obtained with digital microscope MC-40 provided with videocamera. Images were analyzed using video play software. The size of the drop contour was calculated by averaging at least 5 diameters measured on the image of a drop or a wet spot on the leather surface obtained by videorecording of the process with the microscope camera located perpendicular to the material surface. The relative drop contour was used. It was estimated as a ratio of the current value to the initial size. The initial size was determined by extrapolating the initial section of kinetic dependence of contour to the time equaling zero.

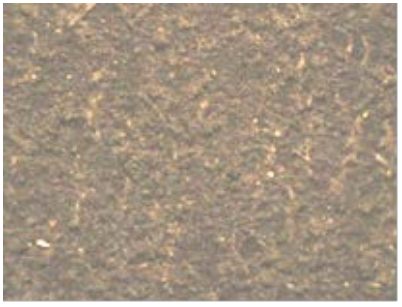

(a)

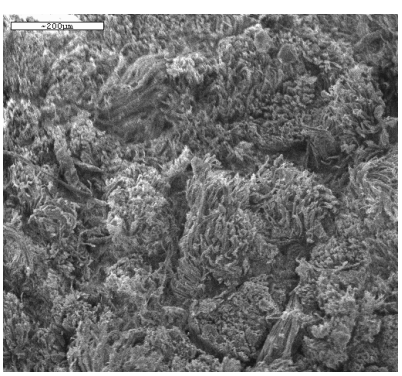

(d)

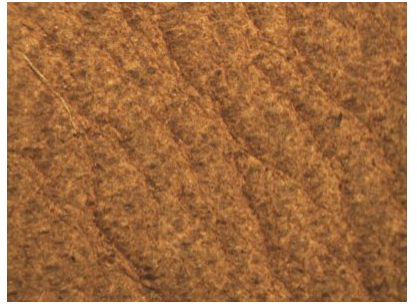

(b)

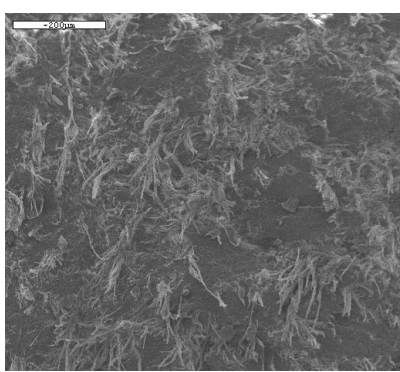

(e)
The surface microstructure was studied with digital stereoscopic microscope DMW143 and scanning electronic microscope Jeol JSM-5300LV.

The mechanical properties of leathers were determined according to test method GOST 938.11-69 [15] on testing machine PT-250M-2. Abrasion resistance test specimens (200 cycles) were done on machine IPK-1 with the load of $25 \mathrm{H}$ on the spindle. During the experiment the spindle rotation direction was measured. Flex resistance test was done on machine IPK-2 using specimens sizing $45 \times 80 \mathrm{~mm}$. The rotation speed was $106 \mathrm{~min}^{-1}$, the number of cycles-25,000. The relaxation properties of leather were studied on testing machine RELAX-M. The specimens sizing $70 \times 70 \mathrm{~mm}$ were used in these tests.

Resistance to the effect of solar radiation and weather was determined according to test method GOST 8979-75 [15] applying the artificial solar radiation-climate unit ADL during 4 hours with a bath temperature of $40^{\circ} \mathrm{C}$ $50^{\circ} \mathrm{C}$.

Such characteristics as roll-off angle, 2-hour water absorption (GOST 938.24-72) and wetting capacity (GOST 8972-78), water uptake (GOST 8972-78), hygroscopic properties and water yielding capacity (GOST 8971-78), water and vapor permeability were determined applying the standard techniques [15].

The equations of functional relationships describing experimental results were made applying the least-square method. The adequacy of the applied mathematical models was confirmed by the Fisher criterion with confidence level 0.95 .

\section{Results and Discussion}

Figure 1 shows surfaces of the studied leathers. In the

Figure 1. Optical ((a)-(c)) and SEM images ((d)-(f)) of surfaces of split ((a), (d)), nubuck ((b), (e)) and velour split ((c), (f)). 
case of split leather the surface structure is inhomogeneous and contains many capillaries sizing not more than 50 - $100 \mathrm{~mm}$, pores, filaments, etc. On the nubuck leather surface the smooth, even areas and the thinned (residual) nap cover are observed. The size of smooth areas is about $1 \times 1 \mathrm{~mm}$; some defects (pores) with the average diameter from 5 to $30 \mu \mathrm{m}$ are visible on the surface.

The surface of velour splits is highly inhomogeneous and contains many capillaries (channels). It should be noted that the surface of the velour splits abounds, apart from pores and capillaries, with villuses that, unlike nubuck, are curved to the surface and form a kind of a net on the leather surface.

Perhaps different morphology of the surface of the materials will influence the kinetics of changes in the water contact angle and the behavior of the drop on the leather surface. Figure 2 shows the dependence of the contact angle of a water droplet on contact time with the split, nubuck and velour split. The observations of the drop behavior lasted not more than $7 \mathrm{~min}$. The contact angle for these materials was more than $90^{\circ}$, which could indicate to their hydrophobic properties. However, the water contact angles decrease with time and the maximum rate of changes of this characteristic was observed in the split, while the minimal rate-in the velour split. After $10 \mathrm{~s}$ the water contact angle for the split equaled $72^{\circ}$. For nubuck the contact angle is less than $90^{\circ}$ in 2 min and its values decreases sharply with further observations. The surface of the velour split maintained its apparent hydrophobic properties to $4 \mathrm{~min}$ and then the water contact angle reached $85^{\circ}$. Consequently, in 4 min the surface of the velour split may be also described as hydrophilic.

During preliminary studies we used only linear approximation in describing the processes of changes in the water contact angle. The process rate was determined as

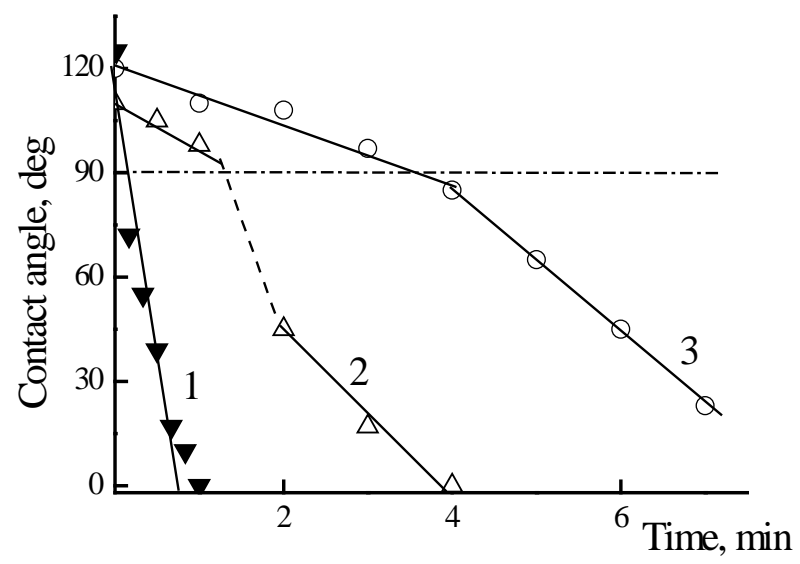

Figure 2. Kinetic dependence of the water contact angle on the surfaces of initial specimens of split (1), nubuck (2) and velour split (3). The dash line marks the water contact angle equaling $90^{\circ}$. a derivative of water contact angle function by time. The rates of changes in the water contact angle on various leathers are given in Table 1 . The spreading and absorption of a drop on the split surface occur rather quickly and at a permanent rate. In case of nubuck and velour split two kinetic areas of changes $\Theta$, characterized by different rates, may be observed. For the nubuck-drop system the rate of decreasing the water contact angle in the first and second area shows nearly a 2-fold difference and for the velour split this difference becomes nearly 4-fold.

Changes of the contact angle in time are connected both with spreading and absorbing of a drop. During the period of observation the drop evaporation may be neglected. The kinetics of drop spreading was estimated by the rate of changes in the contour of a wet area from a water drop on the surface of these materials (Figures 3 and 4). The rates of changes relative diameter of the drop contour are presented in Table 1.

The images in Figure 3(A) show that on the split the drop spreading and absorption are accompanied by capillary flow via microcapillaries (channels) available among the structural elements of the surface: in $10 \mathrm{~s}$ the edges of a wet spot from a drop become uneven and in 60 s, i.e. after absorption is completed, the contours of the wet spot acquire the uncertain shape. The process of spreading is characterized by a constant rate (Table 1 , Figure 4, line 1). Quick spreading and absorbing of a drop permit to conclude that the microstructural elements of the split surface possess the hydrophilic properties.

The drop behavior on the nubuck surface is quite different (Figure 3(B) and Figure 4, line 4). During 1-2 min this material absorbs the drop without visible change in the size of a wet spot contour. The decrease of the water contact angle is also a proof of the drop absorption (Figure 2). Then the contour increases, while the diameter of the drop proper is practically not changed. If at the first stage the absorption rate by the surface layer is obviously greater than the spreading rate, then at the second stage everything is vice versa-the spreading rate exceeds the absorption rate. The behavior of a drop on the

Table 1. Rates of changes in the water contact angles, $\Theta$, and relative sizes of Drop aureoles, $D$, for different Leathers.

\begin{tabular}{ccc}
\hline Material & Rate of changes $\Theta$, degr/min Rate of changes D, $\mathrm{mm}^{-1}$ \\
\hline Split & 115.1 & 1.22 \\
Nubuck & & \\
1 (slow) stage & 12.0 & 0.10 \\
2 (quick) stage & 22.5 & 0.75 \\
Velour split & & \\
1 (slow) stage & 6.3 & 0.00 \\
2 (quick) stage & 23.6 & 0.03 \\
\hline
\end{tabular}




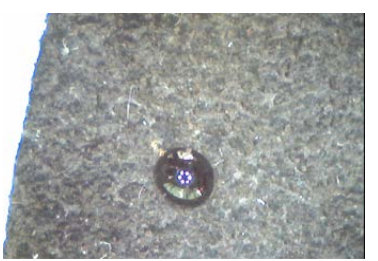

(a)

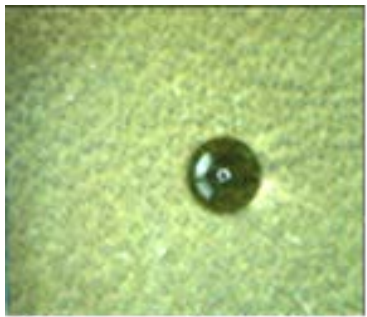

(a)

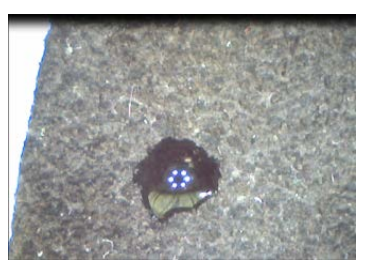

(b)

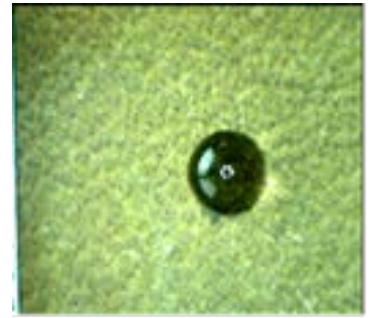

(b)

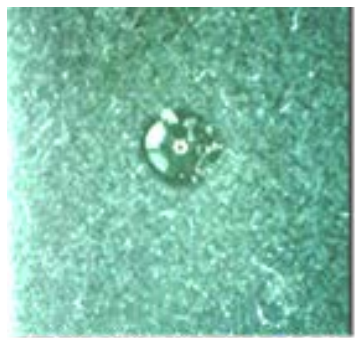

(a)

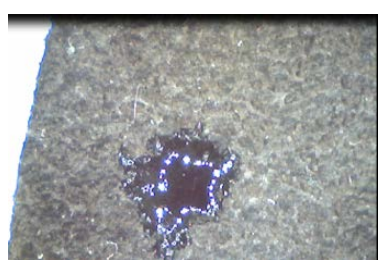

(c)

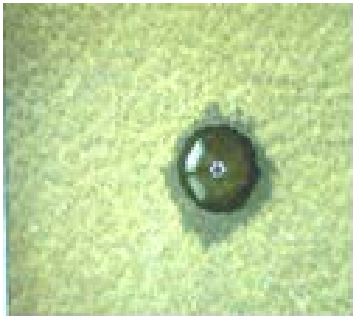

(c)

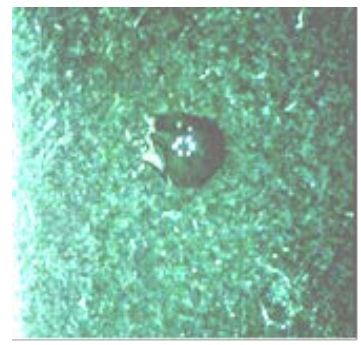

(b)

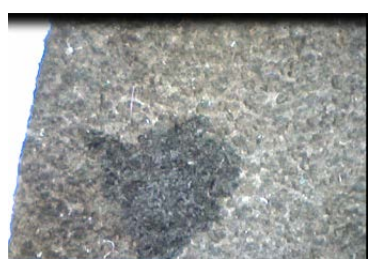

(d)

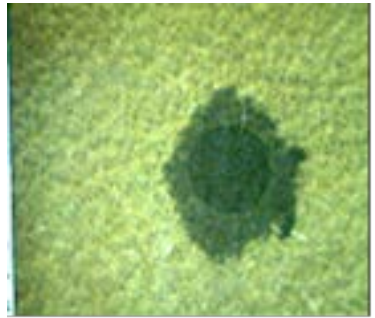

(d)

Figure 3. Behavior of a water drop on the surface of split (A), nubuck (B) and velour split (C) during: (A) - 0 (a), 10 (b), 30 (c) and $60 \mathrm{~s} \mathrm{(d);} \mathrm{(B)-1} \mathrm{(a),} 2$ (b), 3 (d) and $4 \mathrm{~min}$ (d); (C) -2 (a) and $5 \mathrm{~min}$ (b).

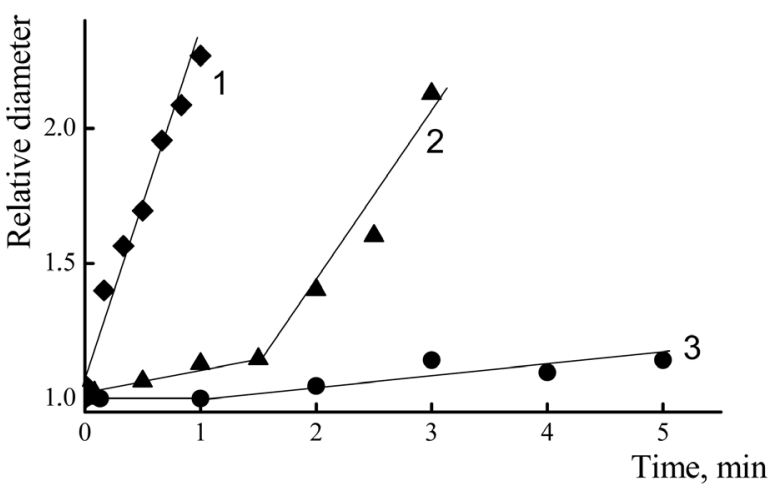

Figure 4. Kinetic curves of changes of the relative drop aureole on the surface of split (1), nubuck (2) and velour split (3).

nubuck surface may be described as reverse wetting of the surface. Accordingly, the dependence of the relative diameter of the drop perimeter, D, on time has two sections where the rate of $\mathrm{D}$ changes differs nearly 7-times (Table 1). Perhaps such specific behavior of a drop is connected with different density of the surface and internal layers of nubuck which, in its turn, is predetermined by the technology of such footwear leather making. The rate of the drop absorbing and spreading on the nubuck surface is visibly less than on split. If for the drop-split system the spreading and absorbing occur during $1 \mathrm{~min}$, then for nubuck-during 4 min.

Observations over the drop behavior on the velour split surface do not show significant changes in the drop perimeter geometry during 3 min (Figure 3(B) and Figure 4, line 3). The insignificant growth of its linear sizes occurs in 3 - 4 min. Perhaps the change of the water contact angle is connected with drop absorption. In this case the drop spreading over the surface is practically not observed (the rate of change of the relative size of a drop contour is near zero). The drop as if "falls down" into the material. The tests of specimens showed that the rate of changes of the relative contour of drop D for such leather in the second section is also minimal.

As it already mentioned above, the surface of the velour split is highly inhomogeneous and contains many capillaries (channels). It seems that such defect structure of the material surface should facilitate quicker absorption of a drop. In fact, quite the opposite is observed. It should be noted that the surface of the velour split, apart from pores and capillaries, abounds in hairs that, unlike, for instance, of nubuck, are curved to the surface and form a kind of a net on the leather surface (Figures 1(c) and (f)). Such peculiar arrangement of nap in the velour split was formed during material manufacturing. It creates 
an air layer between the nap cover and the leather surface that protects the material from quick penetration of water into it. The water drop is absorbed when the structural elements of the net are wetted and soaked.

Therefore, studies of changes of the water contact angle and sizes of the drop contours in time permitted to reveal the specific behavior of the drop on the structurally inhomogeneous surfaces of leathers. On the split the drop spreading and absorbing and, as a result, decrease of the water contact angle, increase of the diameter of the drop aureole occur uniformly; the rate of changes of these characteristics does not alter until complete absorption. The obtained results allow for a conclusion that the homogeneous wetting regime is observed on the split surface. For leathers with polished front surfaces in view of peculiar arrangement of residual nap on the surface (velour split) or density of the leather surface layer (nubuck) the induction periods in the drop spreading and absorbing are usually observed when the changes of the water contact angles and diameters of the drop aureoles are not high. These periods of apparent hydrophobicity, i.e. when the water contact angles are over $90^{\circ}$, probably, describe the heterogeneous wetting regime. Upon the end of this period the rates of the mentioned processes grow sharply and the homogeneous wetting regime is realized. The results of the conducted research prove the hydrophilic nature of the structural elements of natural leathers.

Therefore, the use of split, nubuck and velour split for making the external parts of the footwear uppers is not feasible due to their hydrophilic properties. The additional hydrophobization of their surfaces is required.

Modified leather. The macroscopic analysis of treated leathers shows that the application of hydrophobizator at a rate of $0.4 \mathrm{ml} / \mathrm{dm}^{2}$ does not lead to changes in the leather microstructure, and the surface morphology of treated materials is identical to the original ones.

Treatment of the surface of the studied leathers with the hydrophobic agent permits not only to increase the water contact angle, but to decrease significantly the rate of its changes. For the split the water contact angle is stable for $120 \mathrm{~s}$ and makes $130^{\circ}$ (Figure 5(a)). No changes in the drop aureole geometry or its absorption are observed during this time. Moreover, the applied water drops slide easily over the leather surface. It should be noted that for the initial split the drop was absorbed in 1 $\min$.

The application of hydrophobizators on the surface of ground leathers also results in the growth of the water contact angle and prevents spreading and absorbing of the drop (Figures 5(b) and (c)). Thus, the contact angle of the velour split remains unchanged through the whole observation period ( 7 minutes) and is equal to $130^{\circ}$; the size of the drop aureole does not also change. For nubuck

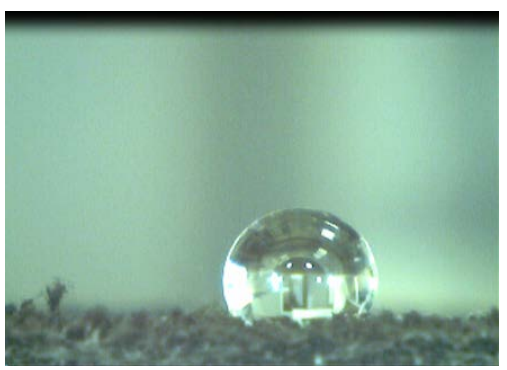

(a)

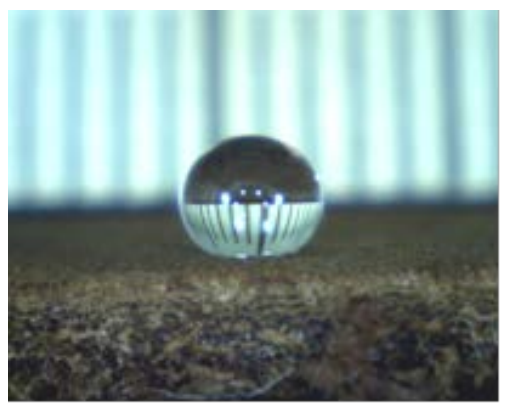

(b)

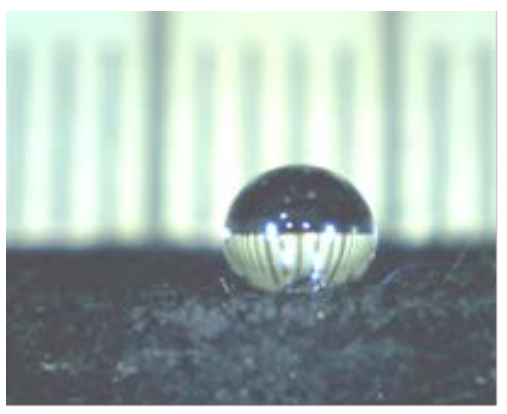

(c)

Figure 5. Photographs of a water dropletes on the surface of the modified with fluoro-containing silane split (a), nubuck (b) and velour split (c). The time of drop staying on the surfaces if 1 (a), 4 (b) and $7 \mathrm{~min}$ (c).

the applied drop also remains stable and is not absorbed during the observed time period (4 min). Regardless of the high value of the water contact angle the water drops do not slide over the surface of the treated specimens of nubuck and velour split, perhaps, due to the presence of nap on these leathers.

As is known, for obtaining the materials with the water contact angles not less than $120^{\circ}$ it is necessary to have the joint effect of the surface roughness and its chemical structure [10-12]. It can be concluded that a high hydrophobic level of modified leathers is associated with the combination of these two factors. The first is the low surface tension of the applied fluoro-containing silane equaling $6 \mathrm{MJ} / \mathrm{m}^{2}$ [10] and the second is the specific morphology of the surface of natural leather. At changing of the chemical structure of the surface of fiber-porous materials it is the latter factor that permits to realize the high and stable water contact angles. If for non-modified 
materials the capillary, fiber-porous structure of leathers is a negative factor facilitating spreading and absorbing of water drops, then after application of fluoro-containing silane and, accordingly, decrease of the surface tension of structural elements of split, ground leathers its effect on the hydrophobic properties of materials becomes positive. It can be asserted that the morphology of the surface of modified leathers allows for realization of the stable heterogeneous wetting regime.

Effect of hydrophobic treatment on physical-mechanical and hygienic properties of split. The effect of hydrophobic agent on the leather properties was studied on the split specimen that originally featured the lowest water resistance among the selected leathers. The split is used widely in manufacturing of everyday footwear. The advantages of this material are its low cost, easy care and good vapor permeability, while among its disadvantages are its low waterproofness and resistance to wear. Regardless of the mentioned disadvantages, the footwear of split is manufactured not only for summer, but also for winter and spring-autumn seasons during which the footwear is subject to the impacts of some negative factors, such as soaking, drying, mechanical loads, etc.

The results presented in Table 2 show that treatment of split with fluoro-containing silane reduces significantly its water absorption and soaking, increases water proofness not affecting, at the same time, its hygroscopic and water uptake values.

Figure 6 shows the relaxation spectra of the studied specimens (dependence of the material pliability, L, $\mathrm{MPa}^{-1}$, on time). The spectrum of the modified split specimen practically coincides with the spectrum of untreated material. Consequently, the surface modification of split with fluoro-containing silane does not change the stress-and-strain and relaxation properties of leather.

The split specimens were subject to multiple bending,

Table 2. Properties of original and surface-modified split.

\begin{tabular}{ccc}
\hline Indicator & Original & Modified \\
\hline The water contact angle ${ }^{*}$, deg & $72 \pm 2$ & $130 \pm 2$ \\
Roll-off angle, deg & $180 \pm 5$ & $70 \pm 5$ \\
Moisture capacity, \% ${ }^{* *}$ & 160 & 116 \\
Soaking, \%*** & 114 & 77 \\
Hygroscopicity, \% & 10 & 11 \\
Water uptake, \% & 9 & 10 \\
Watertightness, s & 2.5 & 5 \\
Vapor permeability, mg/cm ${ }^{*} \cdot \mathrm{hr}$ & 47 & 50 \\
Relative vapor permeability, \% & 70 & 75 \\
\hline
\end{tabular}

*Measurements were conducted in $10 \mathrm{~s}$ after drop application; ${ }^{* *}$ 2-hour moisture capacity; ${ }^{* * *}$ 2-hour soaking.

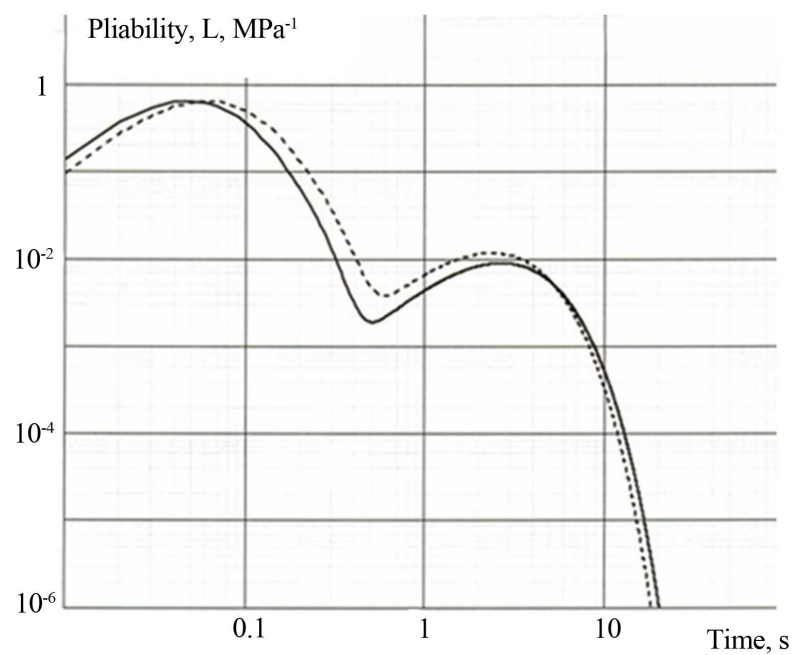

Figure 6. Spectra of relaxation time of original (continuous line) and modified with fluoro-containing silane split (dashed lines).

abrasion, impacts of solar radiation and climate. Table 3 gives the values of the limiting wetting angles after the above tests. For modified specimens this indicator showed not more than $8 \%$ changes. Accordingly, the hydrophobic properties of the treated split are stable to the action of wear factors.

The authors of the paper [13,14] assumed that after application of fluoro-containing silane and subsequent thermal treatment of the specimens the 2-dimensional molecular layer chemically bound with the material surface is formed. The formation of the siloxane bonds (-O-Si-O-) and creation of the oligomeric layer of hydrophobizator help to change the point contacts of individual molecules into multi-centered, which improves the interactions between the formed hydrophobic layer and the leather surface. The formed protective multi-center oligomeric layer based on fluoro-containing silane serves to reduce the effect of the wear factors on morphology of the split surface and maintains its hydrophobic properties.

Summing up the obtained results we can make a conclusion that hydrophobic treatment of split used for making external parts of the footwear uppers with fluorocontaining silane improves the watertightness of leather not affecting its mechanical, relaxation and hygienic properties. The hydrophobic properties of modified split are resistant to the effect of solar radiation and climate, multiple bending and abrasion.

\section{Conclusion}

The microstructure of the leather surface, provided its structural elements possess hydrophilic properties, determines the wetting regime and duration of the induction period of changeover from heterogeneous to homogene- 
Table 3. Effect of wear factors on the water contact angle* of original split and split modified with fluoro-containing silane.

\begin{tabular}{ccc}
\hline Wear factor & Original & Modified \\
\hline- & 72 & 130 \\
Flexure & 65 & 127 \\
Abrasion & 67 & 120 \\
Solar radiation and climate & 70 & 120 \\
\hline
\end{tabular}

*Measurements were conducted in 10 seconds after drop application.

ous wetting. The heterogeneous regime of wetting is distinguished by low rates of spreading and absorbing of water droplets. On the contrary, the homogeneous wetting is characterized by high rates of its spreading and absorbing. A necessary condition of the stable heterogeneous wetting regime and achieving hydrophobicity of leather with structurally inhomogeneous face layer is to preserve the microstructure features of their surface at hydrophobic treatment. The morphological peculiarities of split and ground leather ensure stable hydrophobic properties after application of efficient hydrophobizators.

\section{Acknowledgements}

This work is financially supported by the Federal Target Program "Research and scientific-pedagogical personnel of innovative Russia” (Grant No. 14.B37.21.1088).

\section{REFERENCES}

[1] K. T. Sarkar, "Theory and Practice of Leather Manufacture,” Macmillan India Press, Madras, 1997.

[2] S. Sundar, N. Vijayalakshmi, S. Gupta, R. Rajaram and G. Radhakrishnan, "Aqueous Dispersions of PolyurethanePolyvinyl Pyridine Cationomers and Their Application as Binder in Base Coat for Leather Finishing," Progress in Organic Coatings, Vol. 56, 2006, pp.178-184. http://dx.doi.org/10.1016/j.porgcoat.2006.04.001

[3] E. H. A. Nashy, M. M. Essa and A. I. Hussain, "Synthesis and Application of Methyl Methacrylate/Butyl Acrylate Copolymer Nanoemulsions as Efficient Retanning and Lubricating Agents for Chrome-Tanned Leather,” Journal of Applied Polymer Science, Vol. 124, No. 4, 2012, pp. 3293-3301. http://dx.doi.org/10.1002/app.35208

[4] O. A. Mohamed, A. B. Moustafa, M. A. Mehawed and N. H. El-Sayed, " Styrene and Butyl Methacrylate Copolymers and Their Application in Leather Finishing," Journal of Applied Polymer Science, Vol. 111, No. 3,
2009, pp. 1488-1495.

http://dx.doi.org/10.1002/app.29022

[5] Y. Wang, F. Qiu, B. Xu, J. Xu, Y. Jiang, D. Yang and P. $\mathrm{Li}$, "Preparation, Mechanical Properties and Surface Morphologies of Waterborne Fluorinated PolyurethaneAcrylate,” Progress in Organic Coatings, Vol. 76, No. 5, 2013, pp. 876-883.

[6] K. M. S. Meera, R. M. Sankar, A. Murali, S. N. Jaisankar and A. B. Mandal, "Sol-Gel Network Silica/Modified Montmorillonite Clay Hybrid Nanocomposites for Hydrophobic Surface Coatings," Colloids and Surfaces B: Biointerfaces, Vol. 90, 2012, pp. 204-210. http://dx.doi.org/10.1016/j.colsurfb.2011.10.018

[7] J. Hu, J. Ma and W. Deng, "Properties of Acrylic Resin/Nano-SiO ${ }_{2}$ Leather Finishing Agent Prepared via Emulsifier-Free Emulsion Polymerization," Materials Letters, Vol. 62, No. 17-18, 2008, pp. 2931-2934. http://dx.doi.org/10.1016/j.matlet.2008.01.127

[8] O. Yilmaz, C. N. Cheaburu, G. Gulumser and C. Vasile, "Rheological Behaviour of Acrylate/Montmorillonite Nanocomposite Latexes and Their Application in Leather Finishing as Binders,” Progress in Organic Coatings, Vol. 70, No. 1, 2011, pp. 52-58. http://dx.doi.org/10.1016/j.porgcoat.2010.10.001

[9] S. Smitha, P. Shajesh and P. Mukundan, "Synthesis of Biocompatible Hydrophobic Silica-Gelatin Nano-Hybrid by Sol-Gel Process," Colloids and Surfaces B: Biointerfaces, Vol. 55, No. 1, 2007, pp. 38-43.

[10] L. B. Boinovich and A. M. Emelyanenko, "Hydrophobic Materials and Coatins: Principles of Design, Properties and Applications," Russian Chemical Reviews, Vol. 77, No. 7, 2008, pp. 583-600. http://dx.doi.org/10.1070/RC2008v077n07ABEH003775

[11] M. Nosonovsky, "Multiscale Roughness and Stability of Superhydrophobic Biomimetic Interfaces," Langmuir, Vol. 23, No. 6, 2007, pp. 3157-3161. http://dx.doi.org/10.1021/la062301d

[12] N. A. Patankar, "On the Modeling of Hydrophobic Contact Angles on Rough Surfaces,” Langmuir, Vol. 19, No. 4, 2003, pp. 1249-1253. http://dx.doi.org/10.1021/la026612+

[13] N. V. Evsyukova, M. A. Myshkovskii, L. M. Polukhina O. A. Serenko, L. N. Nikitin and A. M. Muzafarov, “ Making Fabrics Water-Repellent with Fluorine-Containing Silane in Supercritical Carbon Dioxide Medium," Fiber Chemistry, Vol. 41, 2009, pp. 46-52.

[14] N. V. Evsyukova, M. A. Myshkovskii, L. M. Polukhina O. A. Serenko and A. M. Muzafarov, "Effect of Wear Factors on Lyophobic Properties of Fabric Modified by a Fluorosilane,” Fiber Chemistry, Vol. 43, No. 2, 2011, pp. 134-137.

[15] Federal Agency on Technical Regulation and Metrology. http://www.gost.ru 